\title{
1 Two step PCR method to exchange the resistance cassette of a
}

2

$3 \quad$ Nils Cremer and Anne Diehl

4

5 Department of NMR-supported structural Biology, Leibniz-Forschungsinstitut für Molekulare

6 Pharmakologie (FMP), Robert-Rössle-Str. 10, 13125 Berlin, Germany

7

8 Correspondence: $\quad$ diehl@fmp-berlin.de

9 Keywords: ligation independent cloning, exchange antibiotic resistance, protein expression

10 Abstract: 50 words

11 Manuscript: 859 words 


\section{Abstract}

22 For co-transformation of two plasmids, both have to possess different antibiotic selection markers. If

23 that is not the case, normally the gene of interest (GOI) is subcloned into another vector. Here we

24 introduce a fast and easy method to exchange the antibiotic resistance cassette (ARC) in only two

25 PCR steps.

26 Method Summary

27 To shuttle the antibiotic resistance cassette (ARC) from one vector to another, one can amplify the

28 ARC of interest and use the resulting PCR-product as a primer pair for the next amplification step.

29 Simply remove parental DNA template by Dpnl digestion, transform PCR product directly in E. coli

30 cells, select transformants on an appropriate agar plate and isolate target vector by plasmid

31 preparation.

32

33

34

35

36

37 
Progress in structural biology depends on properly folded und functional recombinant proteins. After completion of the big structural genomics projects the low hanging fruits for $E$. coli expression have been almost entirely achieved. The challenge now is to produce difficult-to-express proteins in an improved E. coli environment, as this host is the easiest and cheapest one to handle with respect to NMR-labelling. One possibility is to optimize $E$. coli protein homeostasis by initiation of stress by sigma factor 32 (1). The appropriate plasmid pBAD.Sigma32.I54N (Figure 1A) carrying ampicillin resistance can be obtained via Addgene from Jeff Kelly. Sigma 32 causes the up-regulation of proteostasis network components like chaperones, folding enzymes or proteases. We have used that system surprisingly well for stable expression of an intrinsic unfolded protein with improved yield.

Encouraged by that, we now want to use it for soluble expression of proteins with a high tendency to form inclusion bodies. Unfortunately most of our constructs have ampicillin as the selection marker, making co-transformation with pBAD.Sigma32.154N impossible due to resistance being the same. Instead of re-cloning all our constructs we decided to change the antibiotic resistance cassette (ARC) of pBAD.Sigma32.I54N. A common pET26b vector (69862, Merck, Germany) was chosen as a template for the kanamycin ARC (Figure 1B). Initially both ARCs with their flanking regions were analyzed using alignments with respect to homolog regions in both directions. Indeed a reverse complimentary sequence downstream the ampicillin ARC of pBAD.Sigma32.154N is identical to a sequence upstream the kanamycin ARC of pET26b and vice versa. Therefore two XL primers were synthesized (BioTez, Berlin, Germany) according to Table 1. With these primers the ampicillin ARC downstream of the sigma 32 and with the same translational orientation will be replaced by the kanamycin ARC in the opposite direction (Figure 1C). The reverse orientation is recommended for the kanamycin ARC (2).

The forward primer with 56 nucleotides matches to $100 \%$ in both vectors and the reverse primer with 55 nucleotides fits to $71 \%$ (39 nucleotides underlined part Table 1) to pET26b and $100 \%$ to pBAD.Sigma32.I54N. If those identical stretches do not exist, the XL-primer can be designed by taking in 5'-3' direction 25-30 nucleotides from the ARC flanking site of the target vector (in our case 
pBAD.Sigma32.154N) combined with 25-30 nucleotides of the donor vector (in our case pET26b) to amplify first the ARC of interest and then introduce the resulting PCR product into the target vector via a second PCR. The possibility of changing the orientation of the ARC is then given via a primer and vice versa for the reverse primer.

For both PCR reactions the KOD Hot Start DNA Polymerase Kit (71086, Merck, Germany) was used.

The first PCR with pET26b as template and donor for the kanamycin ARC was performed as follows in a $50 \mu$ set up: $1 x$ KOD buffer; $2 \mathrm{mM} \mathrm{MgSO}_{4} ; 0.2 \mathrm{mM}$ each of the $4 \mathrm{NTP}^{\prime}$; $0.3 \mu \mathrm{M}$ of both primer;

hold

The expected PCR product of about 1000 bp was separated on a $0.8 \%$ agarose gel (Figure 2a) and purified (LSKGEL050, Montage Gel Extraction Kit, Millipore, USA) for use as a primer pair in the final PCR step.

The second PCR to exchange the ampicillin ARC by the amplified kanamycin ARC in pBAD.Sigma32.I54N was set up as the first PCR, but with the double amount of KOD polymerase and $6 \mu \mathrm{l}$ of $1 \mathrm{kB}$ PCR product with $40 \mathrm{ng} / \mu \mathrm{l}$ (final $7.2 \mathrm{nM}$ ) as primers. The cycling program was extended.

\begin{tabular}{llll}
92 & $95{ }^{\circ} \mathrm{C}$ & $5: 00 \mathrm{~min}$ & \\
93 & $94{ }^{\circ} \mathrm{C}$ & $0: 30 \mathrm{~min}$ & \\
94 & $60{ }^{\circ} \mathrm{C}$ & $1: 00 \mathrm{~min}$ & $5 \times$ \\
95 & $68{ }^{\circ} \mathrm{C}$ & $6: 00 \mathrm{~min}$ & \\
\hline
\end{tabular}




$\begin{array}{rlll}96 & 94{ }^{\circ} \mathrm{C} & 0: 30 \mathrm{~min} & \\ 97 & 55^{\circ} \mathrm{C} & 1: 00 \mathrm{~min} & 13 \times \\ 98 & 68{ }^{\circ} \mathrm{C} & 6: 00 \mathrm{~min} & \\ 99 & 68{ }^{\circ} \mathrm{C} & 24: 00 \mathrm{~min} & \\ 100 & 4{ }^{\circ} \mathrm{C} & \text { hold } & \end{array}$

101 After digestion of the template plasmid with $\mathrm{Dpnl}$ at $37^{\circ} \mathrm{C}$ for 1 hour and heat inactivation for $5 \mathrm{~min}$

102 at $80^{\circ} \mathrm{C} 2 \mu$ l of the treated PCR product was used to transform NovaBlue GigaSingles competent cells

103 (71227, Merck, Germany) according to NovaBlue transformation protocol (3). Within 12 to 18 hours

104 transformants grew on kanamycin but not on ampicillin selection plates.

105 In principle this result was proof enough that the kanamycin resistance cassette has been inserted

106 correctly into the target vector. Additionally, using Sanger sequencing (Source Bioscience, UK) we

107 demonstrated that pBAD.Sigma32.154N-kana still carries the gene for Sigma factor.

108 Furthermore we analyzed plasmid preparations of six clones by restriction digest (Figure 2B).

109 The robustness of the method is very good, as the first PCR was successful in a broad window of

110 annealing temperatures, many transformants were produced, six analyzed clones possessed all the

111 expected digestion patterns and code for sigma32. An unwanted CT doubling in the middle of the

112 reverse primer had not influenced the result negatively, as untranslated flanking regions were used

113 as primer pairing positions and some mismatches are tolerated.

\section{Author contributions}

116 N.C. performed the experiments. A.D. design the work, including the primer. N.C and AD wrote the 117 manuscript. 
120 pBAD.Sigma32.I54N was a gift from Jeffery Kelly (Addgene plasmid \# 59982). We thank Catherine L.

121 Worth (FMP) for reading the manuscript.

\section{Competing interests}

The authors declare no competing interests.

References

1. Zhang X, Liu Y, Genereux JC, Nolan C, Singh M and Kelly JW. (2014) The Heat-Shock 
Table 1. Primer for 1. PCR and their corresponding sites in vectors used.

\begin{tabular}{|c|c|c|c|}
\hline primer & sequence & pET26b & pBAD.Sigma32.154N \\
\hline forward & $\begin{array}{l}\text { 5'CTTTGATCTTTTCTACGGGGTCTGACGCTC } \\
\text { AGTGGAACGAAAACTCACGTTAAGG3' }\end{array}$ & $3872-3926$ & $4000-3946$ \\
\hline reverse & $\begin{array}{l}\text { 5'GCGTTTCTACAAACTCTITTGTTTATTTTC } \\
\text { TAAATACATTCAAATATGTATCCGC3' }\end{array}$ & $\underline{4854-4816}$ & $2868-2923$ \\
\hline
\end{tabular}

137

138 Figure 1. Plasmid maps (Vector NTI, Fisher Scientific, UK). A) pBAD.Sigma32.I54N with ampicillin

ARC, B) pET26b donor of the kanamycin ARC und C) the resulting pBAD.Sigma32.I54N-kana. Used

restriction sites are indicated.

Figure 2. Analysis by agarose gels A) Products of 3 parallel set ups for the first PCR with different annealing temperatures (Ta) analyzed in a $1.5 \%$ agarose gel. Lane 1: 18 cycles with Ta $45^{\circ} \mathrm{C}$; Lane 2:

5 cycles with Ta at $45^{\circ} \mathrm{C}$, followed by 13 cycles with Ta of $50^{\circ} \mathrm{C}$; Lane $3: 5$ cycles with Ta at $45^{\circ} \mathrm{C}$,

followed by 13 cycles with Ta of $55^{\circ} \mathrm{C}$; M1: $1 \mathrm{kB}$ Marker (SM0331, Thermo Fisher Scientific) B)

Restriction digest of pBAD.Sigma32.I54N and pBAD.Sigma32.154N-kana analyzed by $0.8 \%$ agarose

147 (4812 bp) digested with BamHI (FD0054) and Smal (FD0663) is only linearized as expected as it possess no cleavage site for Smal; Lane 2a-f: 6 clones of pBAD.Sigma32.154N-kana digested with pBAD.Sigma32.I54N-kana digested with BamHI and Aval (FD0384, cleaves inside the kanamycin 
A

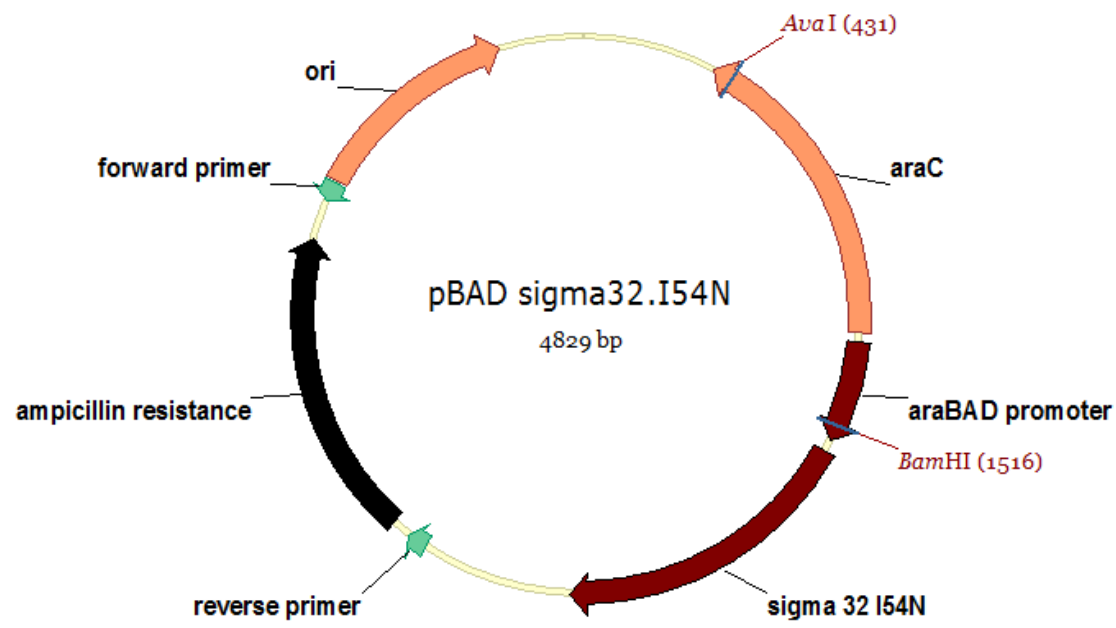

B

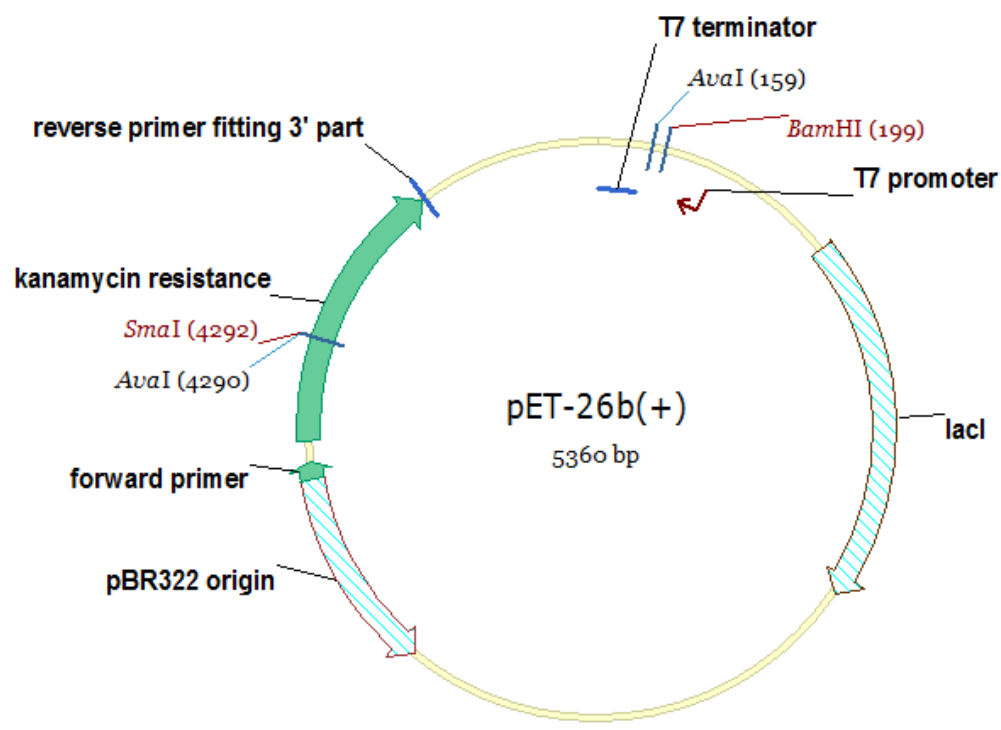

C

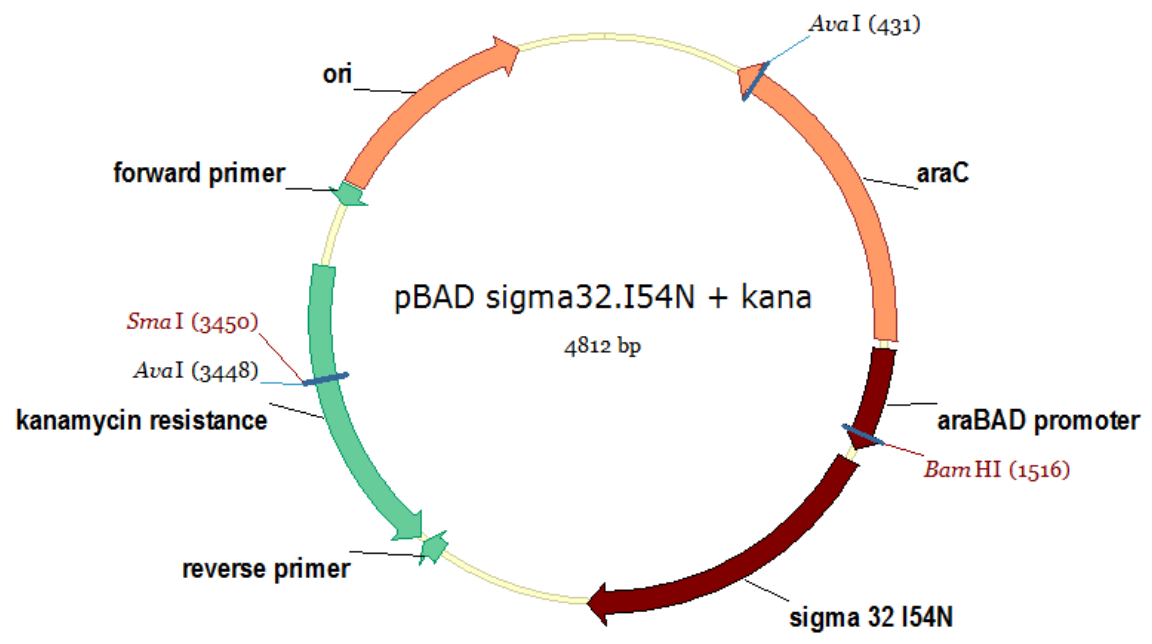


$\begin{array}{lllll}A & 1 & 2 & 3 & M 1\end{array}$

B

$\begin{array}{llllllllllll}M 1 & 1 & 2 a & 2 b & 2 c & 2 d & 2 e & 2 f & M 2 & M 3 & 3 a & 3 b\end{array}$

$\mathrm{bp}$

6000

3000

1000

$-1000$ 3000

$\mathrm{bp}$

1882

925 